\title{
Locus of the effect of specific practice in continuous visual search
}

\author{
WOLFGANG PRINZ \\ Universität Bielefeld, Postfach 8640, 4800 Bielefeld 1, West Germany
}

\begin{abstract}
Specific practice in a visual search task can result either in a selective elaboration of the feature lists of the task stimuli or in a selective emphasis of their status within the task (targets vs. nontargets). According to the first explanation, which is related to the differentiation principle of perceptual learning, specific practice enhances the operation of stimulus identification. According to the second explanation, which is related to the enrichment principle of perceptual learning, it enhances the operation of response selection. Evidence from two transfer experiments with a reversal paradigm argues in favor of the second view, at least for tasks with easily codable letter stimuli. The results are discussed in the framework of a recent model of information integration in visual search.
\end{abstract}

The distinction between stimulus identification and response selection is generally considered as a basic framework for the analysis of performance in speeded classification tasks. The operation of stimulus identification provides a categorization of the stimulus as one of the possible alternatives on the basis of the physical stimulus properties. The operation of response selection then provides a selection of one of the possible responses on the basis of the information associated with the corresponding category (cf. Smith, 1968).

The distinction between stimulus identification and response selection can also be applied to the analysis of performance in continuous visual search tasks. In the first operation; a list item, (e.g., a letter) is identified. In the second operation, information about the item's status is retrieved (target vs. nontarget), and an appropriate response is triggered (stopping the trial in case of a target; taking the next item in case of a nontarget).

As has been frequently demonstrated since Neisser's early experiments (Neisser, 1963; Neisser, Nowick, \& Lazar, 1963), search time is drastically reduced when specific practice with a given task is provided. Stimulus identification and response selection are two possible candidates for the locus of the effect of specific practice.

The efficiency of stimulus identification can be improved because access to the memory representations of the task stimuli becomes selectively adapted to the specific task demands. The selective adaptation of access can imply both selective deletion and selective emphasis of features that mediate the

This study was performed in connection with Grant 2732 from the University of Bielefeld. The author is indebted to Gerd Rübenstrunk and Daniele Paul for their contributions in planning the experiments and discussing their implications. access. Selective deletion pertains to those features which cannot be used for a given discrimination problem because their discriminatory power is low (e.g., the roundness of the target letter $Q$ in a task where the context is constructed from the nontargets SGCU). Selective emphasis pertains to features with a high discriminatory power (the closedness of the target vs. the nonclosed structure of the nontargets).

The efficiency of response selection can be improved because the content of the memory representations becomes selectively adapted to the task demands over practce. The selective adaptation of the content can be brought about by an appropriate organization of its components which stresses those attributes that are needed in the present task (the target status of $Q$ and its power to elicit the stopping response) at the cost of some other qualities which are irrelevant (e.g., its name, its status as a consonant, etc.).

The first of these two explanations makes use of the principles underlying the differentiation theory of perceptual learning (Gibson \& Gibson, 1955). It was put forward by Neisser (1967, ch. 3/4) and Rabbitt $(1964,1967)$. The second explanation is loosely related to the principles underlying the association theory of perceptual learning (Postman, 1955). It was first mentioned by Graboi (1971), who discussed the view that practice enhances the availability of information about the category membership of the stimuli (targets/nontargets) without affecting access to the memory representations where this information is stored.

The present study is an attempt to identify the locus of the practice effect by testing its specificity. If practice causes a selective elaboration of the features that mediate access to the memory representations involved, its effect must be specific to the very target/nontarget combinations used. If it causes 
a selective elaboration of the contents of the memory representations, its effect must be specific to the target and nontarget stimuli used, but not necessarily dependent on the particular combinations.

\section{EXPERIMENT 1}

The reversal learning paradigm, which has mainly been used in the areas of human concept formation (Kendler \& D'Amato, 1955) and animal discrimination learning (Mackintosh, 1970; Sperling, 1965), can be adapted to search tasks to provide a critical test between these two alternatives. When, after some practice with the training task $a / b$ (search for one of the targets from category a among nontargets drawn from category b), the task is reversed (b/a), two different patterns of transfer can be expected. On the one hand, the distinguishing features that have been learned in the training task can still be used in the transfer task. This should lead to a positive transfer to $b / a$ as compared to a control transfer task, $y / x$. On the other hand, the "meanings" of all stimuli (as defined by their status as targets vs. nontargets) are reversed. This should result in a negative transfer. The observed performance in the experimental and the control transfer tasks can therefore be used as an indication of what has been learned in the training task.

\section{Method}

Task. Each subject participated in nine experimental sessions which were arranged on consecutive days, as far as possible. Sessions $1,2,4,6$, and 8 were training sessions. The remaining sessions were transfer sessions. In each training session, the subject practiced with a training task which was constant over the whole practice period. The training sessions lasted for $1 \mathrm{~h}$. During this period, $60-80$ practice trials could be run, depending on subject and session. In each transfer session, four different tasks were presented to each subject. One of them was the training task (say, $a / b)$. The second task was its reversal $(b / a)$. In addition, there were two control tasks, $x / y$ and $y / x$. The transfer sessions comprised eight blocks of eight trials each. Blocks $1,3,5$, and 7 were repetitions of the training task. Blocks $2,4,6$, and 8 were assigned to the four transfer tasks (one of which was identical to the training task). The order of transfer tasks was balanced over transfer sessions within each subject.

Each stimulus category (i.e., a, b, x, y) contained four letters, and each task involved a simultaneous search for one of the four possible targets. All targets were applied about equally often within each session or block, respectively.

Search lists and Equipment. The search lists were letter matrices of 8 (columns) by 64 (rows) symbols. One of the 512 locations was filled with a target item which was drawn at random from the target category. The residual positions were filled with nontargets which were drawn at random from the nontarget category of the corresponding task. All letters were printed in upper case (Siemens T 100). Though the position of the target was basically random within each search list, some serial constraints were imposed on the sequence of search lists in order to improve the random appearance of subsequent trials (for details, see Prinz \& Ataian, 1973).

The lists were presented in a search board. The subject was seated at a table on which the search board was mounted. The search board was tilted towards the subject's frontal parallel plane. The subject started a trial by pressing a button. This released a mechanism which uncovered the search list in a vertical window. The subject scanned the list from top to bottom until he detected the target, which he indicated by pointing to it with a stylus. A microswitch in the stylus stopped the electronic counter which had been started at the beginning of the trial. Search times and errors were recorded.

Subjects and Design. Eight paid subjects were divided into two groups of four. The groups differed with respect to the training task. Group 1 was practiced with VBHC/KRTG. The two experimental transfer tasks were VBHC/KRTG and its reversal, and the two control tasks were XSLD/ZPFQ and its reversal. Group 2 was practiced with XSLD/ZPFQ, with this task and its reversal as experimental, and VBHC/KRTG and its reversal as control transfer conditions (see Table 1). In each transfer session, the order of transfer tasks was balanced over subjects.

Data analysis. A linear regression function relating search time to target position was computed for each transfer block. From this equation, the time needed to scan from the top to the mean position of the list (Row 32) was obtained. This score (M) was preferred over the more common use of the slope of the line because it is virtually unaffected by sample fluctuations in the degree of correlation (see Prinz, 1979). In addition to $M$, the error rates (ER) were computed for each transfer block (errors of omission only; false alarms did not occur).

\section{Results}

The analysis of the search times was carried out in two steps. In the first step, an analysis of variance was computed that distinguished two groups of subjects and four sessions and four tasks within subjects. Sessions and tasks turned out to be highly reliable sources of variations. There was a regular decrease of search time over sessions, ranging from $44.2 \mathrm{sec}$ in the first to $31.3 \mathrm{sec}$ in the last transfer sessions $(F=12.96$, df $=3 / 18, p<.01)$. The task factor (which was treated as a random variable) also influenced the search speed significantly $(F=11.77$, $\mathrm{df}=3 / 18, \mathrm{p}<.01)$. The interaction between groups and tasks was also significant $(F=16.38, \mathrm{df}=3 / 18$, $p<.01)$. In the second step, the effect of the reversal shift was evaluated by means of appropriate comparisons. The corresponding results are summarized in Table 1. For comparisons within groups, the effects of the experimental variables were confounded with task effects. For comparisons within tasks, the critical effects were confounded with group differences. Such confounding could be avoided in the data averaged over all subjects. The most critical comparison pertained to the search times under conditions $b / a$ and $y / x$, as compared to the corresponding difference between $a / b$ and $x / y$. The first difference was completely insignificant $(F=1.04$, df $=1 / 18)$, whereas the second one was highly reliable $(\mathrm{F}=46.35$, $\mathrm{df}=1 / 18, \mathrm{p}<.01)$. The interaction was also significant $(F=30.66$, $\mathrm{df}=1 / 18$, $\mathrm{p}<.01)$. There was no interaction that reflected a change in this pattern of effects over the four transfer sessions $(F<1$, in all cases).

Different results were observed in the error rates. The error rate in the reversed task was considerably 
Table 1

Design and Results of Experiment 1

\begin{tabular}{|c|c|c|c|c|c|c|c|c|c|c|c|}
\hline & \multicolumn{6}{|c|}{ Design } & \multicolumn{5}{|c|}{ Results } \\
\hline & \multicolumn{3}{|c|}{ Unreversed } & \multicolumn{3}{|c|}{ Reversed } & \multicolumn{3}{|c|}{ Unreversed } & \multicolumn{2}{|c|}{ Reversed } \\
\hline & & 1 & 2 & & 1 & 2 & & $\mathbf{M}$ & $\overline{\text { ER }}$ & $M$ & $\overline{\mathbf{E R}}$ \\
\hline $\begin{array}{l}\text { Experimental } \\
\text { Control }\end{array}$ & $\begin{array}{l}a / b \\
x / y\end{array}$ & $\begin{array}{l}\text { VBHC/KRGT } \\
\text { XSLD/ZPFQ }\end{array}$ & $\begin{array}{l}\text { XSLD/ZPFQ } \\
\text { VBHC/KRTG }\end{array}$ & $\begin{array}{l}b / a \\
y / x\end{array}$ & $\begin{array}{l}\text { KRGT/VBHC } \\
\text { ZPFQ/XSLD }\end{array}$ & $\begin{array}{l}\text { ZPFQ/XSLD } \\
\text { KRTG/VBHC }\end{array}$ & $\begin{array}{l}a / b \\
x / y\end{array}$ & $\begin{array}{l}28.4 \\
39.3\end{array}$ & $\begin{array}{r}2.0 \\
18.6\end{array}$ & $\begin{array}{ll}\mathrm{b} / \mathrm{a} & 40.7 \\
\mathrm{y} / \mathrm{x} & 39.1\end{array}$ & $\begin{array}{l}31.9 \\
20.4\end{array}$ \\
\hline
\end{tabular}

Note-Design: Mapping of tasks to conditions for Groups 1 and 2. The training tasks are identical to the unreversed experimental tasks. Results: Mean $M$ scores (in seconds) and mean error rates (in percentages) over all subjects.

higher than in the corresponding control task $(\mathrm{p}<.01$, as indicated by randomization test for matched pairs based on the differences of the absolute error frequencies between conditions $b / a$ and $y / x)$. The error rate in the training task was much lower than in the control task ( $\mathrm{a} / \mathrm{b}$ vs. $\mathrm{x} / \mathrm{y} ; \mathrm{p}<.01$ for the same test). Again, this pattern of error rates was basically repeated within each of the four transfer sessions. Though the overall error rate dropped from $46 \%$ to $24 \%$ between the first and the last transfer session, the relative inferiority of the reversal condition was observed in each session.

\section{Discussion}

These results can be explained in one of two ways. The first explanation postulates that practice affects both the accessibility of the memory representations and the availability of their content. Selective elaboration of the features that mediate access transfers positively, whereas selective learning of the target vs. nontarget status transfers negatively to the reversal task. Under this assumption, the observed equivalence in search speed between the reversal and the control task indicates that these two effects are approximately equal in size and happen to balance each other. The nonequivalence of the corresponding error rates indicates that the negative effect of response reversal is larger than the beneficial effect of selectively elaborated features, as far as errors are concerned. The difference between the speed and the error results is not unreasonable. Search speed is contingent upon the efficiency of target/nontarget discrimination, which depends on both "differentiation" of features and "enrichment" of the content of memory representations. However, the incidence of an error of omissions is perhaps independent of the selectivity of the features that mediate the access to these representations and is only contingent upon the selectivity of their content.

The second explanation postulates that search speed in the reversal task does not reflect transfer effects at all. Instead, it is assumed that the search through the new lists is initially conducted under the control of very recent nontarget information. According to this view, the system integrates and stores information about the nontargets within each particular trial. Each item is then tested against the stored representations of the previous nontargets (cf. Prinz, 1979). In case of a match, it is classified as a nontarget (nontarget recognition). As nontarget recognition is controlled by information that is integrated within the new task, the old target status of the new nontarget items is irrelevant to performance and the reversal and the control task can be scanned with the same speed. However, in case of a mismatch, the item's status can only be identified by making contact to permanently stored information about its status (target identification). Accordingly, the old nontarget status of the new targets is relevant to performance. The old status interferes with the new one-which leads to an increase in the errors of omission as compared to the control condition.

These conclusions are supported from the results of a reversal experiment by Shiffrin and Schneider (1977). When they reversed targets and nontargets after some practice on a noncontinuous search task, the frequency of errors of omission was drastically increased, whereas false alarms were virtually unaffected by the reversal (Figure 3, p. 132). If it is assumed that the omission rate reflects the influence of the old nontarget status of the new targets and that the false alarm rate is related to the old target status of the new nontargets, it must be concluded that the new nontargets are processed in a way that is insensitive to their previous status.

\section{EXPERIMENT 2}

The second experiment was run in order to reach a decision between these two explanations. In addition to the reversal condition, another transfer condition was introduced which was expected to be more sensitive to the possible contributions of a selective elaboration of features. Suppose that a subject is practiced with two search tasks, say $a / b$ and $c / d$, and that, after some time, he is transferred to two transfer tasks which result from crossing over the categories, viz, a/d and $\mathrm{c} / \mathrm{b}$. The exchanged tasks differ from the training tasks with respect to target/ nontarget combinations, but not with respect to the target and nontarget categories used. If the locus of the practice effect resides in the features that mediate 
access to the memory representations, performance in the exchanged tasks should be clearly impaired. This is because the specific elaborations of features that have been established for the training tasks can no longer be used in the transfer tasks. On the other hand, the target vs. nontarget status of all stimuli is unaffected. For this reason, performance should be completely unimpaired if the effect of practice resides in the contents of the memory representations. Consequently, the effects of selective elaboration of features can be directly observed in the exchange condition-if they do exist at all.

Still more information about the underlying mechanisms can be derived from comparing the relative magnitude of the effects of exchange and reversal. These two transfer relations were therefore combined in the present experiment.

\section{Method}

Task and Equipment. There were nine experimental sessions per subject. In the training sessions (Sessions 1, 2, 4, 6, and 8), the subject practiced with two training tasks $(a / b$ and $c / d)$. Eight different tasks were presented in each transfer session (Sessions 3, 5, 7, and 9). They represented four transfer relations: (1) not reversed/not exchanged $(a / b$ and $c / d) ;$ (2) reversed $/$ not exchanged (b/a and $d / c)$; (3) not reversed/exchanged (a/d and $c / b)$; and (4) reversed/exchanged $(d / a$ and $c / b)$. Each stimulus category $(a / b / c / d)$ contained four letters, and the search was simultaneous with respect to the four targets. The training sessions were divided into five pairs of two blocks. The two blocks of each pair were assigned to the two training tasks. Each block contained seven trials on the corresponding task. The transfer sessions were divided into a warm-up phase and a transfer phase. The warm-up phase comprised two blocks with the training tasks. The transfer phase comprised four pairs of two blocks, corresponding to the two tasks in each of the four transfer relations. The order of the four pairs was constant over the four transfer sessions within each subject. In addition to the training and the transfer task, two trials with an irrelevant search task (targets: (]!; nontargets: )(/) were administered between the blocks in all sessions. This was to reduce immediate transfer effects between consecutive tasks. The form of the search lists and the equipment for their presentation was the same as in Experiment 1.

Subjects and Design. There were four groups of four paid female subjects each (Groups I-IV). The same eight tasks were used in each group (see Table 2). Tasks 1-4 and 5-8 were conceived as low vs. high discriminability tasks. The target/nontarget discrimination could be based on the roundness vs. angularity of the stimuli in 5-8, but not in 1-4. As is also shown in Table 2, the groups were balanced with respect to the mapping of the tasks to the transfer relations, resulting in a Latin square of four groups, four transfer relations, and four pairs of transfer tasks. Another Latin square was used to warrant a balance of the order of presentation of the four pairs of blocks over the four subjects within end group.

Data anglysis. Search times and error rates were first obtained for each of the 16 (subjects) $\times 4$ (transfer sessions) $\times 8$ (transfer tasks) $=512$ transfer blocks. The $M$ scores (see Experiment 1) and the absolute number of errors were then averaged over the two blocks within each pair of transfer tasks. These scores could be combined because preliminary analyses had indicated that the effects of the experimental conditions on the two tasks of each pair were virtually equivalent. The resulting 256 values were subjected to an analysis of variance that distinguished transfer sessions, transfer relations, transfer tasks, and groups. The latter three factors formed a Latin square of size 4 (cf. Winer, 1962: $571 \mathrm{pp}$.).

\section{Results}

The effect of the transfer relations was highly significant in both search speed and errors $(F=26.7$ and 77.9, $\mathrm{df}=3 / 36, \mathrm{p}<.01$, in both cases). As was shown by a partition into the effects of reversal and exchange (see Table 3), reversal was a reliable source of variation $(F=9.5$ and 233.6 , $\mathrm{df}=1 / 36$, $p<.01$ ), whereas the effect of exchange was completely insignificant $(F<1)$ in both cases. The interaction between these two factors was insignificant in the search times $(F<1)$, but it approached significance in the error rates $(F=4.23, \mathrm{df}=1 / 36$, $\mathrm{p}<.05)$. However, the simple effects of exchange within the reversal and nonreversal conditions were unreliable throughout.

The search times and the error rates were reduced over the transfer sessions $(F=13.7$ and 8.9 , $\mathrm{df}=$ $3 / 36, p<.01)$. The pattern of the effects of the transfer relations did not change over the sessions, except for a reliable decrease of the size of the effect of reversal on the search times $(F=5.74$, df $=3 / 108$, $\mathrm{p}<.01)$. Though this effect decreased in size, it remained significant even in the last transfer session.

The differences between the error rates of the transfer tasks were also reliable $(F=9.5$, $\mathrm{df}=3 / 36$, $\mathrm{p}<.01$ ), but they were not related to the assumed differences in discriminability between Tasks $1-4 \mathrm{vs.}$ 5-8. In the search times, no significant differences were observed at all. However, an interaction

Table 2

Design of Experiment 2

\begin{tabular}{|c|c|c|c|c|c|c|c|c|}
\hline & \multicolumn{2}{|c|}{$\begin{array}{l}\text { I } \\
\text { (1) EFLH/AZVX } \\
\text { (2) UPRB/OQGC }\end{array}$} & \multicolumn{2}{|c|}{$\begin{array}{c}\text { II } \\
\text { (3) AZVX/EFLH } \\
\text { (4) OQGC/UPRB }\end{array}$} & \multicolumn{2}{|c|}{$\begin{array}{c}\text { III } \\
\text { (5) EFLH/OQGC } \\
\text { (6) UPRB/AZVX } \\
\end{array}$} & \multicolumn{2}{|c|}{$\begin{array}{c}\text { IV } \\
\text { (7) OQGC/EFLH } \\
\text { (8) AZVX/UPRB } \\
\end{array}$} \\
\hline & NR & $\mathbf{R}$ & NR & $\mathbf{R}$ & NR & $\mathbf{R}$ & NR & $\mathbf{R}$ \\
\hline $\begin{array}{l}\text { NE } \\
\mathbf{E}\end{array}$ & $\begin{array}{l}1 / 2 \\
5 / 6\end{array}$ & $\begin{array}{l}3 / 4 \\
7 / 8\end{array}$ & $\begin{array}{l}3 / 4 \\
7 / 8\end{array}$ & $\begin{array}{l}1 / 2 \\
5 / 6\end{array}$ & $\begin{array}{l}5 / 6 \\
1 / 2\end{array}$ & $\begin{array}{l}7 / 8 \\
3 / 4\end{array}$ & $\begin{array}{l}7 / 8 \\
3 / 4\end{array}$ & $\begin{array}{l}5 / 6 \\
1 / 2\end{array}$ \\
\hline
\end{tabular}

Note-I-IV: Experimental groups. (1)-(8): Transfer tasks (targets/nontargets). The two tasks that are assigned to each group were used as the training tasks. The mapping of the tasks to the transfer relations is shown for each group (R/NR: reversed/not reversed; E/NE: exchanged/not exchanged). 
Table 3

Results of Experiment 2

\begin{tabular}{|c|c|c|c|c|c|c|}
\hline & \multicolumn{3}{|c|}{ Search Times } & \multicolumn{3}{|c|}{ Error Rates } \\
\hline & NR & $\mathbf{R}$ & Mean & NR & $\mathbf{R}$ & Mean \\
\hline $\begin{array}{l}\mathrm{NE} \\
\mathrm{E} \\
\text { Mean }\end{array}$ & $\begin{array}{l}30.08 \\
30.33 \\
30.20\end{array}$ & $\begin{array}{l}41.35 \\
39.57 \\
40.46\end{array}$ & $\begin{array}{l}35.73 \\
34.95 \\
35.35\end{array}$ & $\begin{array}{l}4.8 \\
6.8 \\
5.8\end{array}$ & $\begin{array}{l}23.4 \\
21.0 \\
22.2\end{array}$ & $\begin{array}{l}14.1 \\
14.0 \\
14.0\end{array}$ \\
\hline
\end{tabular}

Note-Search times (in seconds) and error rates (in percentages) as a function of transfer relations (see Table 2). Data averaged over tasks and sessions.

between the discriminability of the training tasks and the transfer relations was observed. As shown in an appropriate partition of the interaction between groups and transfer relations, the effect of reversal on search speed tended to be smaller in Groups I and II than in Groups III and IV (F = 4.23, df = $1 / 36, p<.05)$. The same trend was observed in the error rates, but it was insignificant. These results seem to indicate that the delay in search speed due to the reversal is more pronounced after pretraining with high-discriminability training tasks.

\section{Discussion}

These results seem to rule out the first of the two explanations that were considered in the discussion of Experiment 1. Performance in the transfer tasks is critically dependent on the preservation of the target vs. nontarget status of all items, but not on the preservation of the target/nontarget combinations used in the training tasks.

This strongly supports the view that specific practice selectively improves the availability of information about an item's status, and there is virtually no support of a selective elaboration of the features that mediate access to the memory representations where this information is stored. As the second of these two conjectures is based on a failure to reject the null hypothesis (no effect of exchange), it is admittedly weaker than the first one. However, though an effect of exchanging the categories cannot be definitively ruled out, the size of this possible effect can be neglected when compared to the size of the effect of reversing the categories.

The interaction between exchange and reversal does not argue against this view. If the reversal effect is due to a conflict between an item's new and old status, the conflict can be lower for the exchanged as compared to the nonexchanged task. Suppose that the subject was trained with $a / b$ and $c / d$ and is then transferred to b/a (Condition NE/R). In this case, both the new targets and the new nontargets are related to the same old task, viz, $a / b$. When he is transferred to $d / a$ (Condition $E / R$ ), the new targets are related to $\mathrm{c} / \mathrm{d}$ and the new nontargets to $\mathrm{a} / \mathrm{b}$. In the first condition, only one of the training tasks is reactivated by the new task. In the second condi- tion, both training tasks are reactivated. Their mutual interference offers a possible basis for the explanation of the difference in the reversal effects in Conditions $\mathrm{E}$ and NE. The interaction is reliable only in the errors, not in the speed data. This must be expected from the conclusions drawn from Experiment 1 . If the search speed depends on the efficiency of short-term integration of nontarget information, it must be independent of the aftereffects of the training tasks. This is not true of the error rates, which depend on the strength of the aftereffects of the old nontarget status of the new targets.

The difference between the reversal effects of highand low-discriminability training tasks also argues against selective elaboration of feature lists. In the high-discriminability tasks, the differentiation between targets and nontargets can be based on very few physical features. Therefore, if the effect of specific practice resides in the memory access, performance should be less impaired by the reversal of high- than by low-discriminability training tasks. The results are contrary to this expectation. A tentative explanation can be derived from the assumption that the items in the high-discriminability tasks tend to be coded in terms of their common physical features. This implies that the assumed short-term integration procedure integrates feature information instead of item information. Integration of item information can work without specific practice because the memory representations for the letter stimuli are immediately available due to preexperimental learning. Integration of feature information presupposes some specific practice, however, because the appropriate representations of the common features of the nontargets can be established only within the experiment. When the subject is transferred to the reversal, the memory representations of the common features of the new nontargets are not instantaneously available and his performance will be poorer than in the low-discriminability tasks, where coding is at the item level throughout. Note that abstraction of common features does not imply their selective elaboration: Selective abstraction gives a positive weight to common features of equivalent stimuli, whereas selective elaboration gives a negative weight to common features of nonequivalent stimuli. 


\section{CONCLUSION}

The results of the two experiments join in the conclusion that specific practice improves the availability of an item's categorical status without affecting the selectivity of its access to memory. In the course of practice, both the target status of the targets and the nontarget status of the nontargets are learned at the same time. The status of the targets is defined in the instruction and is maintained in memory during the trials. The status of the nontargets is learned implicitly as a by-product of the assumed short-term integration procedure (Prinz, 1979). When a new task is presented where the status of the targets and the nontargets is unchanged, performance is equivalent to that on the training task because the stored status information can be further used. When the categories are reversed, the old target status of the new nontargets is irrelevant to performance, because these items now undergo the shortterm integration procedure. The old nontarget status of the new targets is relevant to performance, however, because the status of these items must now be maintained in permanent memory.

It seems that the differentiation principle is not needed to account for these results. Instead, specific practice "enriches" the memory representations of the task items by improving the availability of information about their status and/or the appropriate response. Results and conclusions are limited to tasks with alphanumerical stimuli, of course. It is not unfeasible to assume that the differentiation principle has better chances with less overlearned stimulus materials.

\section{REFERENCES}

Gibson, J. J., \& Gibson, E. J. Perceptual learning: Differentiation or enrichment? Psychological Review, 1955, 62, 32-41.
GraBoI, D. Searching for targets: The effects of specific practice. Perception \& Psychophysics, 1971, 10, 300-304.

KendleR, H. H., \& D'Amato, M. F. A comparison of reversal shifts and nonreversal shifts in human concept formation behavior. Journal of Experimental Psychology, 1955, 49, 165-174.

Mackintosh, N. J. Attention and probability learning. In D. I. Mostofsky (Ed.), Attention: Contemporary theory and analysis. New York: Appleton-Century-Crofts, 1970.

NEISSER, U. Decision-time without reaction-time: Experiments in visual scanning. American Joumal of Psychology, 1963, 76, 376-385.

NeISSER, U. Cognitive psychology. New York: Appleton-CenturyCrofts, 1967.

Neisser, U., Novick, R., \& LAzAr, R. Searching for ten targets simultaneously. Perceptual and Motor Skills, 1963, 17, 955-961.

Postman, L. Association theory and perceptual learning. Psychological Review, 1955, 62, 438-446.

Prinz, W., \& Atalan, D. Two components and two stages in search performance: A case study in visual search. Acta Psychologica, 1973, 37, 255-277.

PRINZ, W. Integration of information in visual search. Quarterly Journal of Experimental Psychology, 1979, 31, in press.

RABBIT, P. M. A. Ignoring irrelevant information. British Journal of Psychology, 1964, 55, 403-414.

RABBIT, P. M. A. Learning to ignore irrelevant information. American Journal of Psychology, 1967, 80, 1-13.

Shiffrin, R. M., \& Schneider, W. Controlled and automatic human information processing: II. Perceptual learning, automatic attending, and a general theory. Psychological Review, 1977, 84, 127-190.

Smrth, E. E. Choice reaction time: An analysis of the major theoretical positions. Psychological Bulletin, 1968, 69, 77-110.

SPERling, S. E. Reversal learning and resistance to extinction: A review of the rat literature. Psychological Bulletin, 1965, 63, 281-297.

WINER, B. J. Statistical principles in experimental design. New York: McGraw-Hill, 1962.

(Received for publication August 28, 1978; revision accepted December 11,1978 .) 